\title{
On Constitutive Modeling of Arteries
}

\author{
Fuzhang Zhao \\ APD Optima Study, Lake Forest, CA 92630, USA \\ Email: fuzhangzhao@yahoo.com
}

\begin{abstract}
The magic angle of $\theta_{m}=\arctan [(\sqrt{5}+1) / 2] \approx 58.2825^{\circ}$, rather than $\theta=\arccos (1 / \sqrt{3})$ $\approx 54.7356^{\circ}$, has been discovered through theoretical derivations for arteries to accommodate twist buckling optimally. The magic angle matches many published experimental results by others. As byproducts of the derivation, the stable deformation ranges for normal and shear stretches are defined. The anisotropic continuum stored energy (CSE) functional has been used to model the equibiaxial tension tests of porcine thoracic aortas and special simple normal tests of human abdominal aorta aneurysms. In CSE models, constitutive constants are determined by a trial-and-error-on-digit (TED) method and the linear least squares (LLSQ) method combined.
\end{abstract}

Keywords: Arteries, constitutive modeling, experimental tests, magic angle, TED-LLSQ method.

\begin{tabular}{|c|c|}
\hline $\mathbf{A}_{0 i}$ & plane structure tensor in reference configuration \\
\hline AB.CDEFGHI & decimal number with $\mathrm{A}$ for tens digit, $\ldots$, I for ten-millionths digit \\
\hline$B_{p}, C_{p}$ & coefficients of quasi-linear least squares equations \\
\hline C & right Cauchy-Green tensor \\
\hline$c_{1, i}, c_{2, i}, c_{3, i}, c_{4, i}$ & constitutive constants \\
\hline $\mathbf{F}$ & deformation gradient tensor \\
\hline$I_{1}, I_{2}, I_{3}$ & invariants of the right Cauchy-Green tensor \\
\hline$I_{1, i}, I_{2, i}$ & invariant components of the right Cauchy-Green tensor \\
\hline$N$ & number of data pairs \\
\hline $\mathbf{P}_{i}$ & nominal stress component tensor \\
\hline$\left(P_{j k}\right)_{i}$ & nominal stress component tensor in indicial notation \\
\hline $\mathbf{R}$ & rotation tensor \\
\hline $\mathbf{S}_{i}$ & second Piola-Kirchhoff stress component tensor \\
\hline $\mathbf{U}$ & right stretch tensor \\
\hline$X, x$ & position vectors in reference and current configurations \\
\hline$X_{1}, X_{2}, X_{3}$ & Cartesian coordinates of a particle in reference configuration \\
\hline $\begin{array}{l}x_{1}, x_{2}, x_{3} \\
\text { Subscripts }\end{array}$ & Cartesian coordinates of a particle in current configuration \\
\hline$A, a$ & adventitia, anisotropic \\
\hline$b t$ & biaxial tension \\
\hline$e$ & experimental \\
\hline et & equibiaxial tension \\
\hline$f 1, f 2$ & fiber-1, fiber-2 \\
\hline$I, i$ & intima, index for preferred fiber direction \\
\hline$j, k$ & index for three orthogonal directions \\
\hline$M, m$ & media, magic \\
\hline$n$ & number of preferred fiber directions \\
\hline$p$ & index for experimental and theoretical data \\
\hline sn & simple normal \\
\hline ss & simple shear \\
\hline ssn & special simple normal \\
\hline$u t$ & uniaxial tension \\
\hline$v$ & volume \\
\hline$W$ & wall \\
\hline
\end{tabular}




$\begin{array}{ll}1,2,3 & \text { circumferential, axial, and radial directions } \\ \text { Greek Symbols } & \\ \epsilon & \text { error between model and experiment } \\ \eta & \text { thickness ratio } \\ \theta & \text { fiber angle } \\ \kappa & \text { shear stretch or the amount of shear } \\ \lambda & \text { normal stretch } \\ \boldsymbol{\sigma} & \text { Cauchy stress tensor } \\ \Psi_{a} & \text { anisotropic continum stored energy functional } \\ \text { Abbreviations } & \\ \text { A } & \text { abdominal } \\ \text { AAA } & \text { abdominal aorta aneurysm } \\ \text { CI } & \text { common iliac } \\ \text { CSE } & \text { continuum stored energy } \\ \text { DTA } & \text { descending thoracic aorta } \\ \text { ISF } & \text { incompressible stress-free } \\ \text { LLSQ } & \text { linear least squares } \\ \text { MRI } & \text { magnetic resonance imaging } \\ \text { SBT } & \text { soft biological tissue } \\ \text { T } & \text { thoracic } \\ \text { TED } & \text { trial-and-error-on-digit } \\ & \end{array}$

\section{Introduction}

Arterial problems play a significant role in cardiovascular diseases, which are leading causes of morbidity and mortality in many countries. Arteries are blood vessels that carry oxygen and nutrition-rich blood from the heart into organs, tissues, and the rest of the body. The largest arteries are the thoracic and abdominal aortas, which branch into networks of smaller arteries including the arterioles and the capillaries. In a normal physiological state, arterial walls experience circumferential distension and axial extension deformations. With body movement and surgical procedures, however, arteries are often subjected to shortening, twisting, and bending deformations (Fortier, Gullapalli, and Mirshams, 2014) [1].

Arterial walls consist of three constitutional layers: intima, media, and adventitia. In each layer of the arterial walls, helically coiled collagen fibers are dominant structural reinforcements, carrying major loads. The mean helix angles of collagen fibers vary from intima to media and finally to adventitia. Collagen fibers with both positive and negative helix angles are symmetrically paired and optimally oriented with respect to the circumferential direction of arteries. Cylindrical tubes reinforced with paired helical fibers, instead of orthogonal fiber reinforcements, can distend in the circumferential direction, extend in the axial direction, bend with smooth curves, and resist torsion or twisting deformations (Wainwright, 1988) [2]. There is a magic angle for collagen fiber bundles in adventitia layers. Comprehensive information on arteries can be found in the book chapter written by Holzapfel (2008) [3]. Multi-layered cylindrical tubes reinforced with optimally oriented fibers are common structural components found in biology and engineering over a wide range of scales (Goriely and Tabor, 2013) [4].

There has been much effort in searching for the magic angle. The maximum volume confined by a geodesic fiber of finite length is achieved at an angle of $\theta_{v}=\arctan (1 / \sqrt{2}) \approx 35.2644^{\circ}$ in the study of nematode worms by Clark and Cowey (1958) [5]. Wide-ranging applications of magic angle effects have also been found in clinical magnetic resonance imaging (MRI) studies. Dipolar interactions have angular dependence for highly structured molecules like collagen. Dipolar interactions are approximately modulated by the term $3 \cos ^{2} \theta-1$, where $\theta$ is measured from the main magnetic field to a structure direction. The dipolar interaction due to static field vanishes when $3 \cos ^{2} \theta-1=0$ or $\theta=\arccos (1 / \sqrt{3}) \approx 54.7356^{\circ}$ (Erickson, Prost, and Timins, 1993) [6]. Through purely kinematic analyses of simple shear of soft biological tissues with a randomly oriented in-plane fiber, a conditional maximum fiber stretch occurs at the angle of $\theta=\arctan (\sqrt{2}) \approx 54.7356^{\circ}$. The kinematic analyses of simple shear deformations can equally be applied to arterial vessels under torsion (Horgan and Murphy, 2018) [7]. Linear theoretical analyses 
indicate that the magic angle should be greater (Horgan and Murphy, 2018) [8]. Many tissues, including tendons, cartilage, and peripheral nerves, have shown magic angle effects with proton MRI, in which the maximum signal intensity is achieved at the magic angle. Experimental results, however, show that maximum signal intensities have been measured for both flexor tendons and median nerves at the angle of about $60^{\circ}$ instead of $55^{\circ}$ (Bydder et al., 2007) [9]. An optimal determination of the magic angle helps better understand arterial functions, the applications for which hold tremendous clinical and industrial promise.

Constitutive modeling is essential to physiologically, pathologically, and mechanically understanding arterial vessels since mechanical properties relate structures to functions. Constitutive modeling of arteries requires appropriate experimental characterizations and accurate theoretical predictions combined. The most commonly used experimental methods to characterize arteries are distension-extension-torsion tests of intact arterial vessels, uniaxial tension, biaxial tension, and simple normal tests of layer-specific flat specimens. Distension-extension-torsion tests of intact arterial vessels, however, will not be further discussed due to residual stresses in three orthogonal directions. Three types of residual stress in an intact arterial wall are axial residual stress due to axial pre-stretches, radial residual stress due to radially overlaying layers, and circumferential residual stress due to axial pre-bending (Cieslicki, Piechna, and Gambin, 2018) [10]. Invariant-based anisotropic stored energy functionals are usually used, and applications of which make theoretical modelings frame-indifference. Polynomial, power, exponential, and logarithmic functions of invariant components are often used to construct anisotropic stored energy functionals phenomenologically. Constitutive modeling and mechanics of arteries have been reviewed by Humphrey (1995, 2003) [11,12], Holzapfel and Ogden (2010) [13], Di Puccio, Celi, and Forte (2012) [14], Lanir (2017) [15], and Ogden (2017) [16].

Constitutive modelings of arteries will be conducted by the anisotropic CSE functional recently developed by Zhao (2018) [17]. The anisotropic CSE functional reads

$$
\Psi_{a}=c_{1, i} I_{1, i}+c_{2, i} \sqrt{I_{2, i}}+c_{3, i} \frac{I_{1, i}^{c_{4, i}+1}}{I_{3}^{c_{4, i} / 3}}, \quad(i=1,2, \ldots, n),
$$

where the range for subscript $i,(i=1,2, \ldots, n)$, is omitted for the rest of the equations for simplification, $n$ is the number of different preferred fiber directions, the four coefficients, $c_{1, i}, c_{2, i}, c_{3, i}$, and $c_{4, i}$, are constitutive constants to be determined by experimental tests, the three invariants required for following analyses are

$$
I_{1}=\operatorname{tr} \mathbf{C}, \quad I_{2}=0.5\left(I_{1}^{2}-\operatorname{tr} \mathbf{C}^{2}\right), \quad I_{3}=\operatorname{det} \mathbf{C},
$$

the two invariant components are given by

$$
I_{1, i}=\operatorname{tr}\left(\mathbf{C A}_{0 i}\right), \quad I_{2, i}=0.5\left[I_{1} I_{1, i}-\operatorname{tr}\left(\mathbf{C}^{2} \mathbf{A}_{0 i}\right)\right]
$$

and the plane structure tensor for two families of preferred fiber directions with angles $\pm \theta$ is

$$
\mathbf{A}_{0 i( \pm \theta)}=\left(\begin{array}{ccc}
\cos ^{2} \theta & \pm \cos \theta \sin \theta & 0 \\
\pm \cos \theta \sin \theta & \sin ^{2} \theta & 0 \\
0 & 0 & 0
\end{array}\right)
$$

The primary objectives, therefore, are to optimally derive the magic angle, to constitutively model arteries with the anisotropic CSE functional, to briefly evaluate experimental methods for arteries, to newly develop a special solution technique, i.e. the TED-LLSQ method, and to particularly emphasize advantages of the CSE functional for being no isotropic-anisotropic split.

\section{Constitutive Modelings of Arteries}

\subsection{The Magic Angle}

Based on structural analyses of arteries, twist buckling becomes one of the major concerns among the axial extension, axial torsion, circumferential distension, bending, and shortening deformations since 


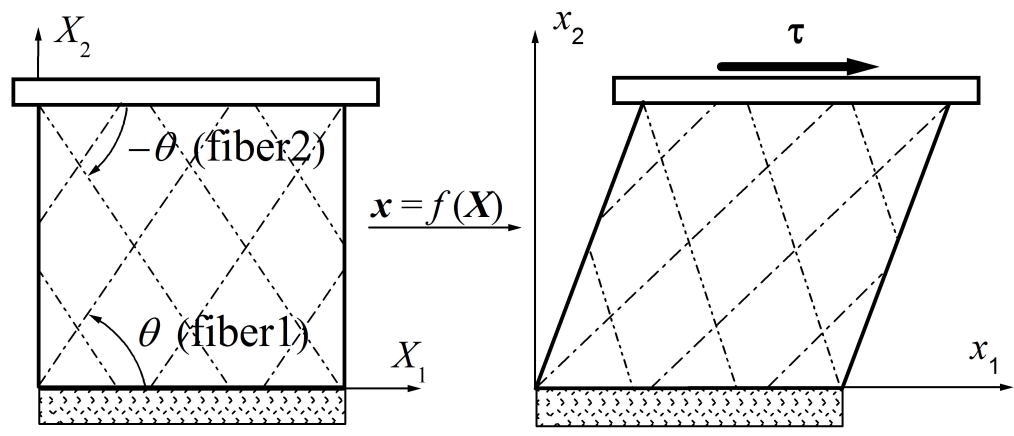

Figure 1. Simple shear tests of unit cube with two families of in-plane fibers.

the energy required for shear deformations is generally less than that needed for normal deformations. Thus, an arterial wall under a simple shear deformation will be kinematically studied, especially on the outermost layer - adventitia - because of largest shear deformations.

The deformation of simple shear can be modeled as

$$
x_{1}=X_{1}+\kappa X_{2}, \quad x_{2}=X_{2}, \quad x_{3}=X_{3},
$$

where the notation of $\kappa=\kappa_{12}$ is used for simplification and $X_{1}, X_{2}, X_{3}$ and $x_{1}, x_{2}, x_{3}$ denote the Cartesian coordinates of a typical particle in reference and current configurations, respectively. In Figure $1, X_{1}$ and $X_{2}$ represent the circumferential and axial directions of arteries, respectively. The angle of shear is $\arctan \kappa$. The deformation gradient tensor $\mathbf{F}_{s s}$ and right Cauchy-Green tensors $\mathbf{C}_{s s}$ and $\mathbf{C}_{s s}^{2}$ are worked out as

$$
\begin{gathered}
\mathbf{F}_{s s}=\left(\begin{array}{ccc}
1 & \kappa & 0 \\
0 & 1 & 0 \\
0 & 0 & 1
\end{array}\right), \\
\mathbf{C}_{s s}=\left(\begin{array}{ccc}
1 & \kappa & 0 \\
\kappa & \kappa^{2}+1 & 0 \\
0 & 0 & 1
\end{array}\right), \\
\mathbf{C}_{s s}^{2}=\left(\begin{array}{ccc}
\kappa^{2}+1 & \kappa^{3}+2 \kappa & 0 \\
\kappa^{3}+2 \kappa & \kappa^{4}+3 \kappa^{2}+1 & 0 \\
0 & 0 & 1
\end{array}\right),
\end{gathered}
$$

where the subscript, ss, represents the simple shear mode.

The three invariants for simple shear deformations are obtained as $I_{1}=I_{2}=\kappa^{2}+3$ and $I_{3}=1$. The corresponding principal stretches can be readily obtained as

$$
\lambda_{1}=\frac{\sqrt{\kappa^{2}+4}+\kappa}{2}, \quad \lambda_{2}=\frac{1}{\lambda_{1}}=\frac{\sqrt{\kappa^{2}+4}-\kappa}{2}, \quad \lambda_{3}=1 .
$$

The two invariant components for angles $\pm \theta$ are given by

$$
\begin{gathered}
I_{1, i( \pm \theta)}=\kappa^{2} \sin ^{2} \theta \pm 2 \kappa \sin \theta \cos \theta+1, \\
I_{2, i( \pm \theta)}=0.5\left(\kappa^{2} \sin ^{2} \theta \pm 2 \kappa \sin \theta \cos \theta+2\right) .
\end{gathered}
$$

In the simple shear deformation, the family of fiber- 1 with angle $\theta$ are in extensional deformations with a fiber stretch $\lambda_{f_{1}}$ while the family of fiber-2 with angle $-\theta$ are in compressive deformations with a fiber stretch $\lambda_{f_{2}}$. The extensional stretch for the family of fiber- 1 of angle $\theta$ is

$$
\lambda_{f_{1}(\theta)}=\sqrt{I_{1, i(\theta)}}=\sqrt{\kappa^{2} \sin ^{2} \theta+2 \kappa \sin \theta \cos \theta+1},
$$


and the compressive stretch for the family of fiber- 2 with angle $-\theta$ is

$$
\lambda_{f_{2}(-\theta)}=\frac{1}{\sqrt{2 I_{2, i(-\theta)}-1}}=\frac{1}{\sqrt{\kappa^{2} \sin ^{2} \theta-2 \kappa \sin \theta \cos \theta+1}},
$$

Taking the derivatives of $I_{1, i( \pm \theta)}$ and $I_{2, i( \pm \theta)}$ with respect to $\theta$, respectively yields

$$
\begin{gathered}
\frac{\partial I_{1, i( \pm \theta)}}{\partial \theta}=2 \kappa\left[\kappa \sin \theta \cos \theta \pm\left(\cos ^{2} \theta-\sin ^{2} \theta\right)\right], \\
\frac{\partial I_{2, i( \pm \theta)}}{\partial \theta}=\kappa\left[\kappa \sin \theta \cos \theta \pm\left(\cos ^{2} \theta-\sin ^{2} \theta\right)\right],
\end{gathered}
$$

and the derivatives (14) and (15) vanish when

$$
\kappa= \pm\left(\frac{\sin ^{2} \theta-\cos ^{2} \theta}{\sin \theta \cos \theta}\right)=\tan ( \pm \theta)-\frac{1}{\tan ( \pm \theta)} .
$$

Substituting $(16)_{1+}$ into $(12)_{2}$ by eliminating $\kappa$, comparing $(9)_{1}$, and simplifying yields many conditional maximum stretches

$$
\lambda_{f_{1}(\theta)}=\tan \theta=\frac{\sqrt{\kappa^{2}+4}+\kappa}{2}=\lambda_{1},
$$

and substituting $(16)_{1-}$ into $(13)_{2}$ by eliminating $\kappa$, comparing $(9)_{2}$, and simplifying yields many conditional minimum stretches

$$
\lambda_{f_{2}(-\theta)}=\frac{1}{\tan (-\theta)}=\frac{\sqrt{\kappa^{2}+4}-\kappa}{2}=\lambda_{2}
$$

It is reasonable to use the reciprocal of shear amount, $1 / \kappa$, to measure shear stability since increasing the amount of shear, $\kappa$, facilitates a twist buckling. For the magic angle, the amount of shear, $\kappa$, needs to be balanced with shear stability, $1 / \kappa$. Rearranging $(16)_{2}$ gives the following fascinating equation

$$
\frac{\kappa}{\tan ( \pm \theta)}=\frac{1}{1+[\tan ( \pm \theta) \kappa]^{-1}}
$$

Since the first dimensionless group $\kappa / \tan ( \pm \theta)$ is inversely related to the second dimensionless group $1 /[\tan ( \pm \theta) \kappa]$, increasing one dimensionless group will decrease the other dimensionless group. The balance between the amount of shear and shear stability can be achieved by equating the two dimensionless groups as

$$
\frac{\kappa_{m}}{\tan \left( \pm \theta_{m}\right)}=\frac{1}{\tan \left( \pm \theta_{m}\right) \kappa_{m}}
$$

Solving (20) for $\tan \left( \pm \theta_{m}\right) \neq 0$ produces the critical shear

$$
\kappa_{m}=1 .
$$

Substituting (21) back into (19) and solving yields the magic angle

$$
\tan \left( \pm \theta_{m}\right)=\frac{\sqrt{5}+1}{2}
$$

or

$$
\pm \theta_{m}=\arctan \left(\frac{\sqrt{5}+1}{2}\right) \approx 58.2825^{\circ} .
$$

Substituting the critical shear (21) into (17) and (18) yields the maximum stable stretch (which also coincides with the maximum stable principal stretch) and the minimum stable stretch (which also coincides with the minimum stable principal stretch), respectively

$$
\lambda_{f_{1}\left(\theta_{m}\right)}=\left(\lambda_{1}\right)_{m}=\frac{\sqrt{5}+1}{2} \approx 1.618
$$




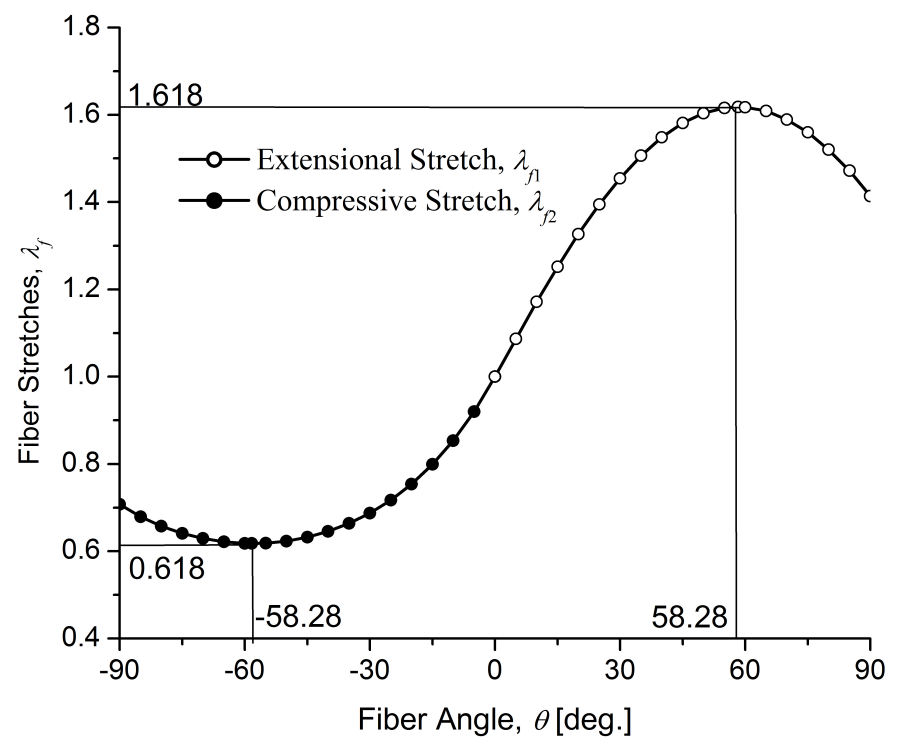

Figure 2. Fiber stretches as function of fiber angle with $\kappa_{m}=1$ in simple shear deformations.

and

$$
\lambda_{f_{2}\left(-\theta_{m}\right)}=\left(\lambda_{2}\right)_{m}=\frac{\sqrt{5}-1}{2} \approx 0.618 .
$$

Thus, it is interesting to see nature's optimal design of the magic angle being equal to the arctangent of the golden ratio $\left(\approx 58.2825^{\circ}\right)$. The maximum stable stretch of fiber- 1 is the golden ratio $(\approx 1.618)$ while the minimum stable stretch of fiber- 2 is the reciprocal of the golden ratio $(\approx 0.618)$. The fiber stretches as a function of fiber angles are also graphically depicted in Figure 2 based on $(12)_{2}$ and $(13)_{2}$ under the condition of $\kappa_{m}=1$.

\subsection{Anisotropic CSE Models for Arteries}

Nominal stress and stretch results are usually calculated from force and extension measurements recorded in experimental tests. Nominal stress component tensor, $\mathbf{P}_{i}$, is related to second Piola-Kirchhoff stress component tensor, $\mathbf{S}_{i}$, by $\mathbf{P}_{i}=\mathbf{S}_{i} \mathbf{F}^{T}=\partial \Psi_{a} / \partial \mathbf{F}$. With the anisotropic CSE functional (1), nominal stress as a function of stretch is

$$
\left(P_{j k}\right)_{i}=\frac{\partial \Psi_{a}}{\partial I_{1, i}} \frac{\partial I_{1, i}}{\partial \lambda_{j k}}+\frac{\partial \Psi_{a}}{\partial I_{2, i}} \frac{\partial I_{2, i}}{\partial \lambda_{j k}}+\frac{\partial \Psi_{a}}{\partial I_{3}} \frac{\partial I_{3}}{\partial \lambda_{j k}}, \quad(j, k=1,2,3) .
$$

The three derivatives of the anisotropic CSE functional (1) for incompressible materials are

$$
\frac{\partial \Psi_{a}}{\partial I_{1, i}}=c_{1, i}+c_{3, i}\left(c_{4, i}+1\right) I_{1, i}^{c_{4, i}}, \quad \frac{\partial \Psi_{a}}{\partial I_{2, i}}=\frac{c_{2, i}}{2 \sqrt{I_{2, i}}}, \quad \frac{\partial \Psi_{a}}{\partial I_{3}}=0 .
$$

Substituting (27), $I_{1, i}=I_{2, i}=I_{3}=1$, and derivatives of invariant components with respect to normal stretches into (26) yields the incompressible stress-free (ISF) condition in reference configuration

$$
2 c_{1, i}+0.5 c_{2, i}+2 c_{3, i}\left(c_{4, i}+1\right)=0 .
$$

Experimental tests of arteries have mainly been conducted by uniaxial tension, biaxial tension, and simple normal tests (also known as constrained biaxial tension tests) under the assumption of incompressibility. Thus, the CSE constitutive models in three deformation modes will be derived based on the equations (2), (3), (4), and (26) through (28). 
Uniaxial Tension Mode. The deformation of uniaxial tension can be modeled as

$$
x_{1}=\lambda_{1} X_{1}, \quad x_{2}=\lambda_{2} X_{2}, \quad x_{3}=\lambda_{3} X_{3} .
$$

With the incompressible constraint of $I_{3}=1$, the related tensors for the uniaxial tension mode in (29) are $\mathbf{F}=\operatorname{diag}\left[\lambda_{1}, \lambda_{2},\left(\lambda_{1} \lambda_{2}\right)^{-1}\right]$ and $\mathbf{C}=\operatorname{diag}\left[\lambda_{1}^{2}, \lambda_{2}^{2},\left(\lambda_{1} \lambda_{2}\right)^{-2}\right]$. The first principal invariant is obtained as $I_{1}=\lambda_{1}^{2}+\lambda_{2}^{2}+\left(\lambda_{1} \lambda_{2}\right)^{-2}$. For the plane structural tensor (4), the two invariant components are

$$
\begin{gathered}
I_{1, i}=\lambda_{1}^{2} \cos ^{2} \theta+\lambda_{2}^{2} \sin ^{2} \theta, \\
I_{2, i}=0.5\left(\lambda_{1}^{2} \lambda_{2}^{2}+\lambda_{1}^{-2} \sin ^{2} \theta+\lambda_{2}^{-2} \cos ^{2} \theta\right),
\end{gathered}
$$

and their derivatives are

$$
\begin{aligned}
\frac{\partial I_{1, i}}{\partial \lambda_{1}}=2 \lambda_{1} \cos ^{2} \theta, & \frac{\partial I_{1, i}}{\partial \lambda_{2}}=2 \lambda_{2} \sin ^{2} \theta \\
\frac{\partial I_{2, i}}{\partial \lambda_{1}}=\lambda_{1} \lambda_{2}^{2}-\lambda_{1}^{-3} \sin ^{2} \theta, & \frac{\partial I_{2, i}}{\partial \lambda_{2}}=\lambda_{2} \lambda_{1}^{2}-\lambda_{2}^{-3} \cos ^{2} \theta .
\end{aligned}
$$

Substituting the derivatives (27), invariant components (30) and (31) and their derivatives (32) and (33) into (26), and converting nominal stresses to Cauchy stresses by $\sigma_{u t, i}=P_{u t, i} \lambda_{i}$ yields the Cauchy stresses, $\sigma_{u t, i}$, for arteries with two identical families of fibers at angles $\pm \theta$

$$
\begin{aligned}
\sigma_{u t, 1}= & 4 c_{1,1} \lambda_{1}^{2} \cos ^{2} \theta+\frac{2 c_{2,1} \lambda_{1}^{2}\left(\lambda_{2}^{2}-\lambda_{1}^{-4} \sin ^{2} \theta\right)}{\sqrt{2\left(\lambda_{1}^{2} \lambda_{2}^{2}+\lambda_{1}^{-2} \sin ^{2} \theta+\lambda_{2}^{-2} \cos ^{2} \theta\right)}}+ \\
& 4 c_{3,1}\left(c_{4,1}+1\right) \lambda_{1}^{2} \cos ^{2} \theta\left(\lambda_{1}^{2} \cos ^{2} \theta+\lambda_{2}^{2} \sin ^{2} \theta\right)^{c_{4,1}}, \\
\sigma_{u t, 2}= & 4 c_{1,2} \lambda_{2}^{2} \sin ^{2} \theta+\frac{2 c_{2,2} \lambda_{2}^{2}\left(\lambda_{1}^{2}-\lambda_{2}^{-4} \cos ^{2} \theta\right)}{\sqrt{2\left(\lambda_{1}^{2} \lambda_{2}^{2}+\lambda_{1}^{-2} \sin ^{2} \theta+\lambda_{2}^{-2} \cos ^{2} \theta\right)}}+ \\
& 4 c_{3,2}\left(c_{4,2}+1\right) \lambda_{2}^{2} \sin ^{2} \theta\left(\lambda_{1}^{2} \cos ^{2} \theta+\lambda_{2}^{2} \sin ^{2} \theta\right)^{c_{4,2}},
\end{aligned}
$$

where the subscript, $u t$, stands for the uniaxial tension mode.

Simple Normal Mode. In simple normal or constrained biaxial tension tests, the circumferential tension test, $\lambda_{1}$ as an independent variable, is conducted with the fixed axial stretch of $\lambda_{2}=1$ while the axial tension test, $\lambda_{2}$ as an argument, is performed under the constrained circumferential stretch of $\lambda_{1}=1$.

Substituting $\lambda_{2}=1$ into (34) and $\lambda_{1}=1$ into (35), respectively, produce the models for simple normal tests

$$
\begin{gathered}
\sigma_{s n, 1}=4 c_{1,1} \lambda_{1}^{2} \cos ^{2} \theta+\frac{2 c_{2,1} \lambda_{1}^{2}\left(1-\lambda_{1}^{-4} \sin ^{2} \theta\right)}{\sqrt{2\left(\lambda_{1}^{2}+\lambda_{1}^{-2} \sin ^{2} \theta+\cos ^{2} \theta\right)}}+ \\
4 c_{3,1}\left(c_{4,1}+1\right) \lambda_{1}^{2} \cos ^{2} \theta\left(\lambda_{1}^{2} \cos ^{2} \theta+\sin ^{2} \theta\right)^{c_{4,1}} \\
\sigma_{s n, 2}=4 c_{1,2} \lambda_{2}^{2} \sin ^{2} \theta+\frac{2 c_{2,2} \lambda_{2}^{2}\left(1-\lambda_{2}^{-4} \cos ^{2} \theta\right)}{\sqrt{2\left(\lambda_{2}^{2}+\sin ^{2} \theta+\lambda_{2}^{-2} \cos ^{2} \theta\right)}}+4 c_{3,2}\left(c_{4,2}+1\right) \lambda_{2}^{2} \sin ^{2} \theta\left(\cos ^{2} \theta+\lambda_{2}^{2} \sin ^{2} \theta\right)^{c_{4,2}},
\end{gathered}
$$

where the subscript, sn, means the simple normal mode.

Alternatively, special simple normal tests with a fixed axial stretch, $\lambda_{2}=1$, are conveniently conducted. The corresponding nominal stress equations in circumferential and axial directions as a function of circumferential stretch, $\lambda_{1}$, are given by

$$
\begin{aligned}
P_{s s n, 1}= & 4 c_{1,1} \lambda_{1} \cos ^{2} \theta+\frac{2 c_{2,1}\left(\lambda_{1}-\lambda_{1}^{-3} \sin ^{2} \theta\right)}{\sqrt{2\left(\lambda_{1}^{2}+\lambda_{1}^{-2} \sin ^{2} \theta+\cos ^{2} \theta\right)}}+ \\
& 4 c_{3,1}\left(c_{4,1}+1\right) \lambda_{1} \cos ^{2} \theta\left(\lambda_{1}^{2} \cos ^{2} \theta+\sin ^{2} \theta\right)^{c_{4,1}}
\end{aligned}
$$




$$
\begin{array}{r}
P_{s s n, 2}=4 c_{1,2} \sin ^{2} \theta+\frac{2 c_{2,2}\left(\lambda_{1}^{2}-\cos ^{2} \theta\right)}{\sqrt{2\left(\lambda_{1}^{2}+\lambda_{1}^{-2} \sin ^{2} \theta+\cos ^{2} \theta\right)}}+ \\
4 c_{3,2}\left(c_{4,2}+1\right) \sin ^{2} \theta\left(\lambda_{1}^{2} \cos ^{2} \theta+\sin ^{2} \theta\right)^{c_{4,2}},
\end{array}
$$

where the subscript, ssn, refers to the special simple normal mode.

Equibiaxial Tension Mode. Having ignored shear and its coupling effects, the simplified equibiaxial tension models can be readily obtained by submitting $\lambda_{1}=\lambda_{2}=\lambda$ into (34) and (35), respectively

$$
\begin{aligned}
& \sigma_{e t, 1}=4 c_{1,1} \lambda^{2} \cos ^{2} \theta+\frac{2 c_{2,1}\left(\lambda^{4}-\lambda^{-2} \sin ^{2} \theta\right)}{\sqrt{2\left(\lambda^{4}+\lambda^{-2}\right)}}+4 c_{3,1}\left(c_{4,1}+1\right) \cos ^{2} \theta \lambda^{2 c_{4,1}+2}, \\
& \sigma_{e t, 2}=4 c_{1,2} \lambda^{2} \sin ^{2} \theta+\frac{2 c_{2,2}\left(\lambda^{4}-\lambda^{-2} \cos ^{2} \theta\right)}{\sqrt{2\left(\lambda^{4}+\lambda^{-2}\right)}}+4 c_{3,2}\left(c_{4,2}+1\right) \sin ^{2} \theta \lambda^{2 c_{4,2}+2},
\end{aligned}
$$

where the subscript, et, indicates the equibiaxial tension mode.

With the ISF condition (28), the CSE models (34) and (35) for uniaxial tension tests, (36) and (37) for simple normal tests or (38) and (39) for special simple normal tests, and (40) and (41) for equibiaxial tension tests can be further simplified in subsequent curve fitting processes.

\subsection{Solution Algorithm for Model Parameters}

One advantage of the anisotropic CSE functional for constitutive modelings is its solution algorithm. For the CSE models, the constitutive constants can be solved by a TED-LLSQ method. One of the equibiaxial tension models is used as an example to illustrate the solution procedure in details.

Substituting (28) into (40), rearranging yields

$$
\sigma_{e t, 1}=c_{2,1}\left[\frac{2\left(\lambda^{4}-\lambda^{-2} \sin ^{2} \theta\right)}{\sqrt{2\left(\lambda^{4}+\lambda^{-2}\right)}}-\lambda^{2} \cos ^{2} \theta\right]+4 c_{3,1}\left(c_{4,1}+1\right) \cos ^{2} \theta\left(\lambda^{2 c_{4,1}+2}-\lambda^{2}\right) .
$$

For normal deformations, the application of the ISF condition gives $c_{1,1}=-0.25 c_{2,1}-c_{3,1}\left(c_{4,1}+1\right)$. The remaining constitutive constants, $c_{2,1}, c_{3,1}$, and $c_{4,1}$, are solved by the TED-LLSQ method. The quasi-linear least squares equations are

$$
\begin{aligned}
c_{2,1} \sum_{p=1}^{N} B_{p}^{2}+c_{3,1} \sum_{p=1}^{N} B_{p} C_{p} & =\sum_{p=1}^{N} \sigma_{e, p} B_{p}, \\
c_{2,1} \sum_{p=1}^{N} C_{p} B_{p}+c_{3,1} \sum_{p=1}^{N} C_{p}^{2} & =\sum_{p=1}^{N} \sigma_{e, p} C_{p},
\end{aligned}
$$

where $\sigma_{e, p}$ is the $p^{\text {th }}$ data of experimental Cauchy stresses and the coefficients for equibiaxial tension tests given by (42) are

$$
\begin{gathered}
B_{p}=\frac{2\left(\lambda_{p}^{4}-\lambda_{p}^{-2} \sin ^{2} \theta\right)}{\sqrt{2\left(\lambda_{p}^{4}+\lambda_{p}^{-2}\right)}}-\lambda_{p}^{2} \cos ^{2} \theta, \\
C_{p}=4\left(c_{4,1}+1\right) \cos ^{2} \theta\left(\lambda_{p}^{2 c_{4,1}+2}-\lambda_{p}^{2}\right) .
\end{gathered}
$$

The error between the CSE model and the experiment is defined by the following equation

$$
\epsilon=\frac{\sqrt{\sum_{p=1}^{N}\left(\sigma_{p}-\sigma_{e, p}\right)^{2}}}{\sqrt{\sum_{p=1}^{N}\left(\sigma_{e, p}\right)^{2}}}
$$

where $\sigma_{p}$ is the $p^{\text {th }}$ data of theoretical Cauchy stresses calculated from $\sigma_{e t, 1}(42)$. 


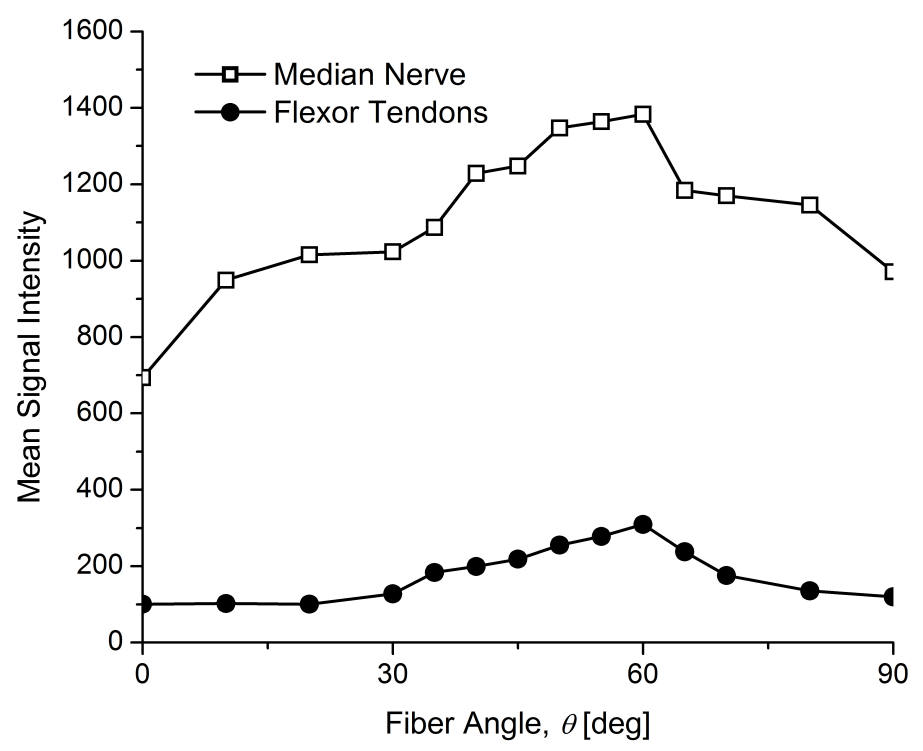

Figure 3. The signal intensity versus fiber angle for flexor tendons and median nerve [9].

The nonlinear constant, $c_{4,1}$, in (46) has to be initially determined in order to solve the equations (43) and (44) linearly. Since $c_{4,1}$ is the one and only one nonlinear constitutive constant, a TED method is specially developed for the anisotropic CSE functional.

For $c_{4,1}=$ AB.CDEFGHI $\in(0,100)$ as an example, the TED method starts with tens digit, i.e., $\mathrm{A}=\{10,20,30,40,50,60,70,80,90\}$. In the nine trials, the number with the smallest error defined in (47) is obtained as A. The number of trials on each of the following digit is 18 instead of 9 since the current number, $\mathrm{AB}$, could be either larger or smaller than the previous number, $\mathrm{A}$, and a zero digit has been previously tested. Then, there are eighteen trials for $\mathrm{B}= \pm 10^{-1} \mathrm{~A}=\{ \pm 1, \pm 2, \pm 3, \pm 4, \pm 5, \pm 6, \pm 7, \pm 8, \pm 9\}$ while adding $\mathrm{AB}$ together. Then, for $\mathrm{C}=10^{-1} \mathrm{~B}=\{ \pm 0.1, \pm 0.2, \pm 0.3, \pm 0.4, \pm 0.5, \pm 0.6, \pm 0.7, \pm 0.8, \pm 0.9\}$ eighteen trials is conducted while adding up to AB.C. A similar process will continue to be used for sequentially smaller digits until the desired digit. In this example, the last desired digit is the tenmillionths $\mathrm{I}=10^{-7} \mathrm{~B}$ while the final decimal number AB.CDEFGHI is constructed and solved with only 153 trials. Thus, the TED-LLSQ method is numerically accurate, stable, and efficient. Furthermore, possible multiple solutions for exponential stored energy functionals, due to ill-conditioned minimization problems, have to be dealt with by trials on multiple initial values of model parameters.

\section{Experimental Results and Models}

\subsection{Experimental Results of the Magic Angle}

The mean signal intensity as a function of fiber angle provides the characteristic signature of the magic angle effect. The signal intensity versus fiber angle for flexor tendons and median nerve published in [9] is digitized and replotted in Figure 3. The mean signal intensities for both flexor tendons and median nerve increase from $0^{\circ}$ up to a maximum at $60^{\circ}$ instead of $55^{\circ}$ and decrease toward $90^{\circ}$.

In addition, fiber angles of aortas and arteries can also be experimentally measured. Fiber angles, $\theta_{I}$, $\theta_{M}$, and $\theta_{A}$, for intimal, medial and adventitial layers of human aorta respectively were measured by Holzapfel (2006) [18]. Layer-specific fiber angles for thoracic (T) and abdominal (A) aortas, and common iliac (CI) arteries, later, have been experimentally measured. Samples were taken from seven locations T1, T2, T3, A1, A2, A3, and CI (Schriefl et al., 2011) [19]. The layer-specific fiber angles, $\theta_{I}, \theta_{M}$, and $\theta_{A}$ 
Table 1. Measured fiber angles and wall thickness ratios of human arteries.

\begin{tabular}{|c|c|c|c|c|c|}
\hline Name & $\theta_{I}\left(^{\circ}\right)$ & $\theta_{M}\left({ }^{\circ}\right)$ & $\theta_{A}\left({ }^{\circ}\right)$ & $\eta_{I} / \eta_{M} / \eta_{A}$ & $\theta_{W}\left(^{\circ}\right)$ \\
\hline Aorta-A & $\pm 18.8 \pm 8.2$ & $\pm 37.8 \pm 20.6$ & $\pm 58.9 \pm 14.8$ & $13 / 50 / 37$ & $\pm 43.1 \pm 16.8$ \\
\hline Aorta- $\mathrm{T}$ & $\pm 40.3 \pm 22.6$ & $\pm 27.5 \pm 15.3$ & $\pm 51.9 \pm 17.0$ & $12 / 57 / 31$ & $\pm 36.6 \pm 16.7$ \\
\hline Aorta-A & $\pm 37.8 \pm 23.7$ & $\pm 24.9 \pm 14.7$ & $\pm 48.9 \pm 18.8$ & $16 / 50 / 34$ & $\pm 35.1 \pm 17.5$ \\
\hline Artery-CI & $\pm 43.7 \pm 24.3$ & - & $\pm 53.8 \pm 17.9$ & $14 / 44 / 42$ & - \\
\hline
\end{tabular}

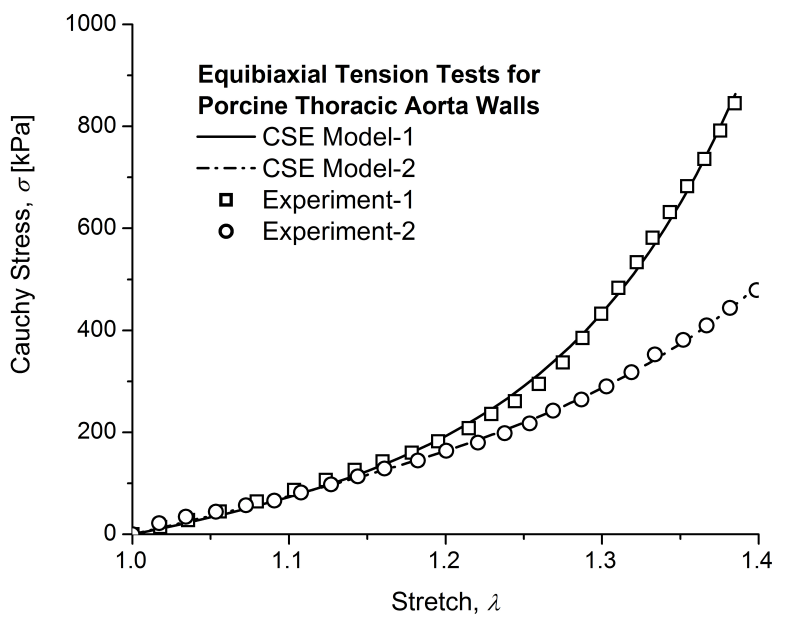

Figure 4. Comparison between CSE models and equibiaxial tension tests for porcine aortas.

and intact wall mean fiber angles, $\theta_{W}$, with standard deviations, and the layer-specific wall thickness ratios, $\eta_{I}, \eta_{M}$, and $\eta_{A}$, are averaged in similar type but different locations and summarized in Table 1.

\subsection{Modeling for Porcine Aorta Walls in Equibiaxial Tension Tests}

Equibiaxial tension tests of porcine descending thoracic aorta (DTA) walls with cruciform specimens have been conducted by Ahuja et al. (2018) [20]. Experimental curves for mid true lumen wall of porcine 5 in both circumferential and axial directions have been used to fit the equibiaxial tension CSE models (40) and (41). A self-developed graphics digitizer with MATLAB has been used to read out pairs of data, and axial Cauchy stress as a function of stretch up to 1.4 has been selected. For thoracic aortas, the mean angle pairs are $\pm 40.3^{\circ}, \pm 27.5^{\circ}$, and $\pm 51.9^{\circ}$ for intima, media, and adventitia, respectively and the corresponding wall thickness ratios are $0.12,0.57$, and 0.31 , respectively [19]. The fiber angle pair of $\pm \theta_{W}=36.6^{\circ}$ is averaged among the angles of intimal, media, and adventitia layers before curve fittings. Two sets of constitutive constants have been solved by the TED-LLSQ method and listed in Table 2 . The comparison between the anisotropic CSE model and the equibiaxial tension test data of the porcine thoracic aorta wall is shown in Figure 4.

\subsection{Modeling for Human Abdominal Aorta Aneurysms in Special Simple Normal Tests}

Special $\left(\lambda_{2}=1\right)$ simple normal tests of human abdominal aorta aneurysm (AAA) walls with cruciform specimens have been conducted by Pancheri et al. (2017) [21]. Experimental curves of AAA averaged over thirteen patients in both circumferential and axial directions have been used to fit the special simple normal CSE models (38) and (39). Fifteen pairs of data, similarly, have digitally been read out. The fiber 


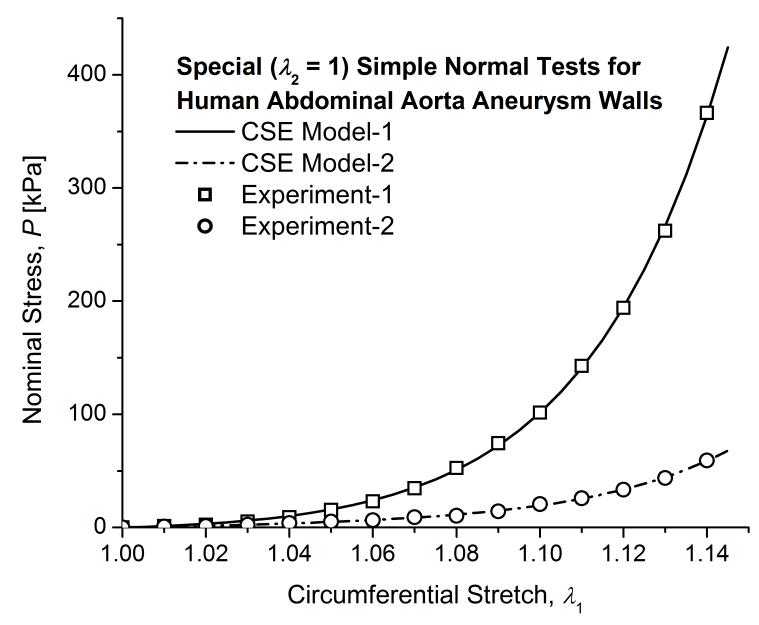

Figure 5. Comparison between CSE models and special simple normal tests for human AAAs.

Table 2. Constitutive constants of CSE models for different aortas.

\begin{tabular}{ccccc}
\hline Aortas & $c_{1, i}(\mathrm{kPa})$ & $c_{2, i}(\mathrm{kPa})$ & $c_{3, i}(\mathrm{kPa})$ & $c_{4, i}$ \\
\hline Porcine DTA-1 & -44.91993 & 165.52322 & 0.53354 & 5.6332578 \\
Porcine DTA-2 & -40.79526 & 150.78283 & 0.52009 & 4.9595702 \\
\hline Human AAA-1 & -23.74536 & 90.66609 & 0.06064 & 16.7908264 \\
Human AAA-2 & -15.68429 & 26.07469 & 0.48364 & 17.9511897 \\
\hline
\end{tabular}

angle pair of $\pm \theta_{W}=6.7^{\circ}$ is adopted before curve fittings. Two sets of constitutive constants have been solved by the TED-LLSQ method and listed in table 2. A comparison of the anisotropic CSE model and the special simple normal test data of human AAA walls is shown in Figure 5.

\section{Discussion}

\subsection{Fiber Angles of Aortas}

The magic angle of $\theta_{m}=\arctan [(\sqrt{5}+1) / 2] \approx 58.2825^{\circ}$ has been derived based on kinematic analyses of simple shear deformations. Direct histological image measurements for mean angles of adventitial layers listed in Table 1 match the theoretical magic angle. Indirect MRI measurements of mean signal intensity for both flexor tendons and median nerve clearly demonstrate that the magic angle is closer to $60^{\circ}$ rather than $55^{\circ}$ shown in Figure 3. The adventitial layer of aortas is reinforced with collagen fibers oriented at the symmetrically paired magic angles. Only a fiber in tension can support the same maximum load regardless of fiber lengths while a fiber in compression, bending, and torsional deformations can not. Thus, the main function of an adventitia is to accommodate twist buckling, in which shear stretches are efficiently converted to normal stretches of fibers.

The mean angles for intact aorta walls, $\theta_{W}$, estimated and listed in the last column of Table 1, are very close to the angle of $\theta_{v}=\arctan (1 / \sqrt{2}) \approx 35.2644^{\circ}$. Under $\theta_{v}$ with certain geodesic fiber length, the lumen volume is maximized. Inversely, the fiber length oriented at $\theta_{v}$ is minimized for certain lumen volume. Thus, aorta walls with the mean fiber angle of $\theta_{v}$ may minimize the usage of collagen fibers.

The mean angle for human abdominal aorta walls is about $35.1^{\circ}$ while that of human AAA is only about $6.7^{\circ}$. In the wall of a pressurized artery, the circumferential stress is twice the axial stress. As 
abdominal aortas grow into aneurysms, shears from both solid and fluid are reduced but circumferential tensions become major issues. More collagen fibers, therefore, are needed for abdominal aorta aneurysms than abdominal aortas.

\subsection{Experimental Tests of Arteries}

Stretches are defined in a general deformation gradient tensor F. For general three-dimensional deformations, $\mathbf{F}$ can be formally and conveniently expressed as

$$
\mathbf{F}=\frac{\partial \boldsymbol{x}}{\partial \boldsymbol{X}}=\left(\begin{array}{lll}
\lambda_{11} & \kappa_{12} & \kappa_{13} \\
\kappa_{21} & \lambda_{22} & \kappa_{23} \\
\kappa_{31} & \kappa_{32} & \lambda_{33}
\end{array}\right)=\left(\begin{array}{lll}
\lambda_{1} & \kappa_{1} & \kappa_{3} \\
\kappa_{2} & \lambda_{2} & \kappa_{5} \\
\kappa_{4} & \kappa_{6} & \lambda_{3}
\end{array}\right) .
$$

In the nine stretches of a deformation gradient tensor, diagonal stretches, $\lambda_{1}, \lambda_{2}$, and $\lambda_{3}$, are normal stretches, giving length change information while off-diagonal stretches, $\kappa_{1}, \kappa_{2}, \kappa_{3}, \kappa_{4}, \kappa_{5}$, and $\kappa_{6}$, are shear stretches, providing angle change information.

Rigid-body translation is automatically removed from the partial differentiations in $\mathbf{F}(48)_{1}$. Rigid-body rotation is also removed in the right Cauchy-Green tensor, in which $\mathbf{C}=\mathbf{F}^{T} \mathbf{F}=\mathbf{U}^{T} \mathbf{R}^{T} \mathbf{R} \mathbf{U}=\mathbf{U}^{2}$ in terms of right stretch tensor $\mathbf{U}$ and rotation tensor $\mathbf{R}$ based on the polar-decomposition theorem [22]. In experimental tests of certain modes, rigid-body motions should be avoided even though they have essentially been removed. For stable deformations in experimental tests of arteries, the normal and shear stretches based on (24), (25), and (21), should be controlled by the following guidelines

$$
0.618 \leq \lambda \leq 1.618 \text { and } \quad-1 \leq \kappa \leq 1
$$

The test results for both DTA and AAA walls are stable data since their maximum deformations follow the guidelines (49). The deformation gradient tensor can clearly capture key features of experimental tests with different deformation modes.

Uniaxial Tension Tests. In uniaxial tension tests of incompressible anisotropic materials, only diagonal stretches exist and the deformation gradient tensor becomes a diagonal tensor of $\mathbf{F}_{u t}=$ $\operatorname{diag}\left[\lambda_{1}, \lambda_{2},\left(\lambda_{1} \lambda_{2}\right)^{-1}\right]$. In uniaxial tension tests of arteries, both circumferential and axial stretches, $\lambda_{1}$ and $\lambda_{2}$, have to be measured simultaneously in order to characterize the anisotropic materials correctly.

The uniaxial tension test is one of the most commonly used tests, having advantages of efficiency, accuracy, and stability over a large deformation range. Physiological deformations of arteries, however, naturally exhibit the biaxial stretching mode of $\lambda_{1} \geq 1$ and $\lambda_{2} \geq 1$, which is beyond the domain of uniaxial tension modes of either $\lambda_{1} \geq 1$ and $\lambda_{2} \leq 1$, or $\lambda_{1} \leq 1$ and $\lambda_{2} \geq 1$. Structural integrity disturbed at lateral edges during uniaxial tension tests of aortas has been observed. As a result of material instabilities, measurements capture uncertainties (Holzapfel, 2006) [18]. Thus, uniaxial tension tests are not appropriate to characterize arteries.

Biaxial Tension Tests. In biaxial tension tests of incompressible anisotropic materials, both diagonal and off-diagonal stretches exist and the deformation gradient tensor becomes

$$
\mathbf{F}_{b t}=\left(\begin{array}{ccc}
\lambda_{1} & \kappa_{1} & 0 \\
\kappa_{2} & \lambda_{2} & 0 \\
0 & 0 & \lambda_{3}
\end{array}\right)
$$

where $\lambda_{3}=\left(\lambda_{1} \lambda_{2}-\kappa_{1} \kappa_{2}\right)^{-1}$.

The biaxial tension test involves one of the most complicated deformations among other available tests because of the number of independent variable stretches. In general, four variables, $\lambda_{1}, \lambda_{2}, \kappa_{1}$, and $\kappa_{2}$, have to be measured. In biaxial tension tests of soft biological tissues (SBT), normal stretches generally range in $\lambda \in[0.7,1.7]$ and shear stretches are limited to $\kappa \in[-0.1,0.1]$ (Potter et al., 2018) [23]. When protocols with constant ratios of $\lambda_{1}$ to $\lambda_{2}$ are used, the independent variable stretch can be reduced, especially for equibiaxial tension tests with $\lambda_{1}=\lambda_{2}$. Stress-stretch curves in biaxial tension tests of 
square specimens are generally overestimated and shear stretches are generally two order of magnitude smaller than normal stretches, i.e. $\kappa \approx 10^{-2} \lambda$ (Aydin et al., 2017) [24]. Cruciform specimen should be used in order to reduce shear stretches and to achieve more uniform force distributions.

The specimen size of about $4 \mathrm{~mm}$ by $4 \mathrm{~mm}$ has been used to deal with issues of arterial sizes, material homogeneity, and accuracy of biaxial tension tests by Potter et al. (2018) [23].

Simple Normal Tests. In simple normal tests, the circumferential tension, $\lambda_{1}$, was conducted under the fixed axial stretch of $\lambda_{2}=1$ while the axial tension, $\lambda_{2}$, was conducted under the fixed circumferential stretch of $\lambda_{1}=1$. In simple normal tests of incompressible anisotropic materials, the deformation gradient tensor becomes

$$
\mathbf{F}_{s n, 1}=\left(\begin{array}{ccc}
\lambda_{1} & 0 & 0 \\
0 & 1 & 0 \\
0 & 0 & \lambda_{1}^{-1}
\end{array}\right) \quad \text { and } \quad \mathbf{F}_{s n, 2}=\left(\begin{array}{ccc}
1 & 0 & 0 \\
0 & \lambda_{2} & 0 \\
0 & 0 & \lambda_{2}^{-1}
\end{array}\right)
$$

Simple normal tests of rabbit abdominal skins were first conducted by Lanir and Fung (1974) [25]. The test was later used to examine if material parameters of strain energy function are physically realistic by Humphrey (1999) [26]. The simple normal test of arteries was further recommended by Veljković and Kojić (2010) [27]. For anisotropic materials including arteries, simple normal tests have only one independent variable $\lambda_{1}$ or $\lambda_{2}$, which are simpler than uniaxial tension tests of two independent variables $\lambda_{1}$ and $\lambda_{2}$. Furthermore, simple normal tests overcome arterial instabilities due to non-physiological deformation modes including uniaxial tension tests.

In simple normal tests, shear stretches can be minimized, and forces needed in fixed directions do not have a stress work-done on materials. Both simple shear and simple normal tests of arteries have only one independent variable despite more complicated loading conditions. Thus, simple normal tests of layer-specific arterial walls are promising.

\subsection{On Isotropic-Anisotropic Split}

SBTs including arteries as fiber-matrix composite materials are usually recognized as anisotropic hyperelastic materials. Many phenomenological constitutive models for arteries additively decompose a stored energy functional into isotropic and anisotropic parts. The concept of the isotropic-anisotropic split might be simply adopted from constitutive models for composite materials with infinitesimal deformations.

In constitutive modeling of anisotropic hyperelastic materials, division in a geometrical sense is not worthwhile since putting parts back as a whole is more difficult than dividing a whole into parts and new interfaces are created after splitting. Interface issues are usually harder to resolve than those of individual parts, let alone the problems of modeling the physical contact between two bodies have not been solved satisfactorily (Podio-Guidugli, 2018) [28].

In constitutive modeling of fiber-matrix composite materials with infinitesimal deformations, fiber and matrix are individually characterized by experimental tests. It is daunting to characterize collagen fiber and liquid-like matrix for arteries individually. Experimental characterizations for arteries under finite deformations, therefore, are conducted on whole fiber-matrix composite materials.

The accuracy on stress magnitudes separately contributed by isotropic and anisotropic parts of a stored energy functional at same stretches in curve-fittings of experimental test results of whole SBT materials is questionable. Generally, the larger difference of stress contributions between isotropic and anisotropic parts transfers to corresponding tangent stiffness matrices. The ill-conditioned stiffness matrix occurs since the unbalance between decomposed isotropic and anisotropic parts of a stored energy functional (Duong, Nguyen, and Staat, 2015) [29].

In the anisotropic CSE functional, anisotropic and uniform deformations, and mean fiber angles are kept as key essences while other geometrical details of fiber, matrix, and even interface are purposely ignored. Since isotropic finite deformations are special cases of anisotropic finite deformations, the anisotropic CSE functional alone is enough to model SBTs and the isotropic part of a stored energy functional is redundant. 


\section{Conclusions}

The magic angle of $\theta_{m}=\arctan [(\sqrt{5}+1) / 2] \approx 58.2825^{\circ}$ has been optimally obtained based on kinematic analyses of simple shear of a unit cube with two symmetrically paired families of fiber bundles. Experimental results from both direct histological image and indirect MRI mean signal intensity show that the magic angle is closer to $58.2825^{\circ}$ rather than $54.7356^{\circ}$. Thus, the major function of adventitial layers of aortas with fiber bundles oriented at the magic angles is discovered to accommodate twist buckling optimally. As byproducts from the magic angle study, the stable deformation ranges for normal and shear stretches are defined.

Unlike exponential-typed stored energy functionals, the anisotropic CSE functional has only one nonlinear constitutive constant. A TED method is newly developed to initially determine the nonlinear constant $c_{4, i}$ so that the rest of constants can be determined by the standard LLSQ method. For a seven-decimal place accuracy, only 153 trials are needed for $c_{4, i} \in(0,100)$, making curve-fittings with excellent numerical properties of stability, efficiency, and accuracy. In addition, the anisotropic CSE functional, without the isotropic-anisotropic split, avoids the ill-conditioned stiffness matrix.

The anisotropic CSE constitutive models for uniaxial tension, biaxial tension, and simple normal tests have been derived. The constitutive constants for the equibiaxial tension tests of porcine DTAs and the special simple normal tests of human AAAs have been solved by the TED-LLSQ method. Simple normal tests, along with simple shear tests, are strongly recommended for characterization of intrinsic and layer-specific mechanical responses of arterial vessels.

\section{References}

1. A. Fortier, V. Gullapalli, and R. A. Mirshams, "Review of biomechanical studies of arteries and their effect on stent performance," IJC Heart \& Vessels, vol. 4, pp. 12-18, 2014.

2. S. A. Wainwright, Axis and Circumference: The Cylindrical Shape of Plants and Animals. Cambridge, MA: Harvard University Press, 1988.

3. G. A. Holzapfel, "Collagen in arterial walls: Biomechanical aspects," in Collagen: Structural and Mechanics, M. K. Franklin, L. C. H. Hui, and D. S. Wong, Eds. New York: Springer, 2008, pp. 285-324.

4. A. Goriely and M. Tabor, "Rotation, inversion and perversion in anisotropic elastic cylindrical tubes and membranes," Proceedings of the Royal Society A, vol. 469, pp. 20130011 (1-19), 2013.

5. R. B. Clark and J. B. Cowey, "Factors controlling the change of shape of certain nemertean and turbellarian worms," Journal of Experimental Biology, vol. 35, no. 4, pp. 731-748, 1958.

6. S. J. Erickson, R. W. Prost, and M. E. Timins, "The "magic angle" effect: Background physics and clinical relevance," Radiology, vol. 188, no. 1, pp. 23-25, 1993.

7. C. O. Horgan and J. G. Murphy, "On the fiber stretch in shearing deformations of fibrous soft materials," Journal of Elasticity, vol. 133, no. 2, pp. 253-259, 2018.

8. - " "Magic angles and fibre stretch in arterial tissue: Insights from the linear theory," Journal of the Mechanical Behavior of Biomedical Materials, vol. 88, pp. 470-477, 2018.

9. M. Bydder, A. Rahal, G. D. Fullerton, and G. M. Bydder, "The magic angle effect: A source of artifact, determinant of image contrast, and technique for imaging," Journal of Magnetic Resonance Imaging, vol. 25, pp. 290-300, 2007.

10. K. Cieslicki, A. Piechna, and W. Gambin, "Two-layered analytical model of arterial wall with residual stress under physiological loading," Applied Mathematical Modelling, vol. 57, pp. 52-63, 2018.

11. J. D. Humphrey, "Mechanics of the arterial wall: Review and directions," Critical Reviews in Biomedical Engineering, vol. 23, no. 1-2, pp. 1-162, 1995.

12. - "Continuum biomechanics of soft biological tissues," Proceedings of the Royal Society of London A, vol. 459, pp. 3-46, 2003.

13. G. A. Holzapfel and R. W. Ogden, "Constitutive modelling of arteries," Proceedings of the Royal Society A, vol. 466, pp. 1551-1597, 2010.

14. F. Di Puccio, S. Celi, and P. Forte, "Review of experimental investigations on compressibility of arteries and the introduction of a new apparatus," Experimental Mechanics, vol. 52, pp. 895-902, 2012.

15. Y. Lanir, "Multi-scale structural modeling of soft tissues mechanics and mechanobiology," Journal of Elasticity, vol. 129 , no. 1-2, pp. 7-48, 2017.

16. R. W. Ogden, "Nonlinear continuum mechanics and modeling the elasticity of soft biological tissues with a focus on artery walls," in Biomechanics: Trends in Modeling and Simulation, Studies in Mechanobiology, Tissue Engineering and Biomaterials, G. A. Holzapfel and R. W. Ogden, Eds. Switzerland: Springer International Publishing, 2008, vol. 20, pp. 83-156. 
17. F. Z. Zhao, "Anisotropic continuum stored energy functional solved by Lie group and differential geometry," Advances in Pure Mathematics, vol. 8, pp. 631-651, 2018.

18. G. A. Holzapfel, "Determination of material models for arterial walls from uniaxial extension tests and histological structure," Journal of Theoretical Biology, vol. 238, pp. 290-302, 2006.

19. A. J. Schriefl, G. Zeindlinger, M. David, D. M. Pierce, P. Regitnig, and G. A. Holzapfel, "Determination of the layer-specific distributed collagen fibre orientations in human thoracic and abdominal aortas and common iliac arteries," Journal of the Royal Society Interface, pp. 0727 (1-12), 2011.

20. A. Ahuja, J. N. Noblet, T. Trudnowski, B. Patel, J. F. Krieger, S. Chambers, and G. S. Kassab, "Biomechanical material characterization of stanford type-b dissected porcine aortas," Frontiers in Physiology, vol. 9, pp. 1317 (1-13), 2018.

21. F. Q. Pancheri, R. A. Peattie, N. D. Reddy, T. Ahamed, W. Lin, T. D. Ouellette, D. Mark, M. D. Iafrati, and A. L. Dorfmann, "Histology and biaxial mechanical behavior of abdominal aortic aneurysm tissue samples," ASME Journal of Biomechanical Engineering, vol. 139, pp. 031002 (1-12), 2017.

22. G. A. Holzapfel, Nonlinear Solid Mechanics: A Continuum Approach for Engineering. Chichester: John Wiley \& Sons, 2000.

23. S. Potter, J. Graves, B. Drach, T. Leahy, C. Hammel, Y. Feng, A. Baker, and M. S. Sacks, "A novel small-specimen planar biaxial testing system with full in-plane deformation control," ASME Journal of Biomechanical Engineering, vol. 140, pp. 051001 (1-18), 2018.

24. R. C. Aydin, S. Brandstaeter, F. A. Braeu, M. Steigenberger, R. P. Marcus, K. Nikolaou, M. Notohamiprodjo, and C. J. Cyron, "Experimental characterization of the biaxial mechanical properties of porcine gastric tissue," Journal of the Mechanical Behavior of Biomedical Materials, vol. 74, pp. 499-506, 2017.

25. Y. Lanir and Y. C. Fung, "Two-dimensional mechanical properties of rabbit skin. II. experimental results," Journal of Biomechanics, vol. 7, pp. 171-182, 1974.

26. J. D. Humphrey, "An evaluation of pseudoelastic descriptors used in arterial mechanics," ASME Journal of Biomechanical Engineering, vol. 121, pp. 259-262, 1999.

27. D. Veljković and M. Kojić, "Prediction of planar uniaxial and constrained biaxial state of deformation by commonly used anisotropic constitutive models in arterial mechanics," Journal of the Serbian Society for Computational Mechanics, vol. 4, pp. 54-74, 2010.

28. P. Podio-Guidugli, "On the mechanical modeling of matter, molecular and continuum," Journal of Elasticity, 2018. [Online]. Available: https://doi.org/10.1007/s10659-018-9709-y

29. M. T. Duong, N. H. Nguyen, and M. Staat, "Physical response of hyperelastic models for composite materials and soft tissues," Asia Pacific Journal on Computational Engineering, vol. 2, pp. 3 (1-18), 2015. 